\title{
Kidenga: Public engagement for detection and prevention of Aedes-borne viral diseases
}

\author{
Chris Schmidt" ${ }^{\star 1}$, Alba Phippard ${ }^{3}$, Jennifer M. Olsen ${ }^{2}$, Kathy Wirt ${ }^{2}$, Andrea Rivera', \\ Adam Crawley ${ }^{2}$, Steve Waterman ${ }^{3}$ and Kacey Ernst ${ }^{1}$
}

${ }^{1}$ Epidemiology and Biostatistics, University of Arizona, Tucson, AZ, USA; ${ }^{2}$ Skoll Global Threats Fund, San Francisco, CA, USA;

${ }^{3}$ Centers for Disease Control \& Prevention (CDC), Atlanta, GA, USA

\section{Objective}

(1) Early detection of Aedes-borne arboviral disease; (2) improved data on Ae aegypti and Ae. albopictus distribution in the United States (U.S.); and (3) education of clinicians and the public.

\section{Introduction}

Zika, chikungunya, and dengue have surged in the Americas over the past several years and pose serious health threats in regions of the U.S. where Ae aegypti and Ae. albopictus mosquito vectors occur. Ae. aegypti have been detected up to 6 months of the year or longer in parts of Arizona, Florida, and Texas where mosquito surveillance is regularly conducted. However, many areas in the U.S. lack basic data on vector presence or absence. The Zika, dengue, and chikungunya viruses range in pathogenicity, but all include asymptomatic or mild presentations for which individuals may not seek care. Traditional passive surveillance systems rely on confirmatory laboratory testing and may not detect emergent disease until there is high morbidity in a community or severe disease presentation. Participatory surveillance is an approach to disease detection that allows the public to directly report symptoms electronically and provides rapid visualization of aggregated data to the user and public health agencies. Several such systems have been shown to be sensitive, accurate, and timelier than traditional surveillance. We developed Kidenga, a mobile phone app and participatory surveillance system, to address some of the challenges in early detection of day-biting mosquitoes and Aedes-borne arboviruses and to enhance dissemination of information to at-risk communities.

\section{Methods}

Kidenga sends a weekly push notification prompting users to report symptoms, travel history, and day-biting mosquito activity. If an individual reports through Kidenga that they or a family member have had symptoms consistent with Zika, dengue, or chikungunya, they receive an email with educational information about the diseases, prevention strategies, and treatment/testing information for clinicians. Upon registration, users can opt in to have additional follow-up via email. At any time, users may also view maps of aggregated user reports, confirmed case counts by county from public health partners (in pilot areas), Aedes distribution maps, information about prevention and control strategies, and news on the diseases and vectors from a curated newsfeed. Users in select pilot areas may also receive press releases issued by their state or local public health department related to the diseases and their vectors. University of Arizona owns and maintains the app and its data. Local and state health departments that want more detailed information on user symptoms and mosquito activity may request and monitor the data at no cost. A marketing campaign to recruit a broad user base is being implemented in Arizona, Texas, and Florida.

\section{Results}

Kidenga was developed with significant input from public health stakeholders and launched in September 2016, accompanied by English and Spanish radio public service announcements in select
Arizona markets, press releases, and a social marketing campaign. A Spanish version of the app is under development. We will describe the results of user registration and survey submissions, challenges identified during development and deployment of this novel surveillance system, plans for data use and evaluation, and collaborations with public health partners.

\section{Conclusions}

The utility of Kidenga as a surveillance system will depend on broad and consistent participation among diverse user populations, particularly in low-risk areas; strategies to integrate health reports for high-risk populations who may not have smartphones; validation of data and development of sensitive and specific algorithms for taking public health action, and buy-in from public health departments to use the data and advocate for this novel surveillance tool. Kidenga's secondary function as an education tool on Aedes-borne viruses is less dependent upon a large user base and can be evaluated separately. Participatory surveillance systems that specifically monitor Aedesborne pathogens are relatively new, and the challenges associated with their early detection may differ from those of other diseases.

\section{Keywords}

participatory surveillance; mosquito; arbovirus; m-Health; Zika

\section{Acknowledgments}

We acknowledge the collaboration of the Arizona Department of State Health Services, City of Laredo Health Department, City of McAllen Health Department, and Florida Department of Health.

\section{${ }^{*}$ Chris Schmidt}

E-mail: cschmidt@email.arizona.edu 